\title{
Volterra composition operators from generally weighted Bloch spaces to Bloch-type spaces on the unit ball
}

\author{
Haiying Li*, Haixia Zhang \\ College of Mathematics and Information Science, Henan Normal University, Xinxiang 453007, P.R. China. \\ Dedicated to George A Anastassiou on the occasion of his sixtieth birthday \\ Communicated by Professor R. Saadati
}

\begin{abstract}
Let $\varphi$ be a holomorphic self-map of the open unit ball $\mathbb{B}, g \in H(\mathbb{B})$. In this paper, the boundedness and compactness of the Volterra composition operator $T_{g}^{\varphi}$ from generally weighted Bloch spaces to Bloch-type spaces are investigated.(C)2012 NGA. All rights reserved.
\end{abstract}

Keywords: Volterra composition operator; generally weighted Bloch space; boundedness; compactness. 2010 MSC: Primary 47B38.

\section{Introduction and preliminaries}

Let $\mathbb{B}$ be the unit ball in $\mathbb{C}^{n}$ and $H(\mathbb{B})$ the class of all holomorphic functions on $\mathbb{B}$. Let $z=\left(z_{1}, z_{2}, \cdots, z_{n}\right), w=$ $\left(w_{1}, w_{2}, \cdots, w_{n}\right)$ be points in $\mathbb{C}^{n}$ and $\langle z, w\rangle=\sum_{j=1}^{n} z_{j} \overline{w_{j}}$. Let $\mathcal{R} f(z)=\sum_{j=1}^{n} z_{j} \frac{\partial f}{\partial z_{j}}(z)$ be the radial derivative of $f \in H(\mathbb{B})$, see for more details in [14].

For any $0<\alpha<\infty$, we define the generally weighted Bloch space $\mathcal{B}_{\log }^{\alpha}$ of holomorphic functions such that

$$
\|f\|_{\mathcal{B}_{\log }^{\alpha}}=|f(0)|+\sup _{z \in \mathbb{B}}\left(1-|z|^{2}\right)^{\alpha}|\mathcal{R} f(z)| \log \frac{4}{1-|z|^{2}}<\infty .
$$

\footnotetext{
${ }^{*}$ Corresponding author

Email address: tslhy2001@yahoo.com.cn (Haiying Li)
} 
When $\alpha=1$, for the case of the unit disk the logarithmic Bloch space has appeared for the first time in characterizing of the multipliers of the Bloch space in [1], for more details in [12] and [13]. In [3], [4] and [5], we studied composition operator on generally weighted Bloch spaces.

A positive continuous function $\mu$ on the interval $[0,1)$ is called normal $([8])$ if there are $\delta \in[0,1)$ and a and $\mathrm{b}, 0<a<b$ such that

$$
\begin{aligned}
& \frac{\mu(r)}{(1-r)^{a}} \text { is decreasing on }[0,1) \text { and } \lim _{r \rightarrow 1} \frac{\mu(r)}{(1-r)^{a}}=0 ; \\
& \frac{\mu(r)}{(1-r)^{b}} \text { is increasing on }[0,1) \text { and } \lim _{r \rightarrow 1} \frac{\mu(r)}{(1-r)^{b}}=0 .
\end{aligned}
$$

If we say that a function $\mu: \mathbb{B} \rightarrow[0, \infty)$ is normal, then we will also assume that $\mu(z)=\mu(|z|), z \in \mathbb{B}$.

The Bloch-type space $B_{\mu}(\mathbb{B})$ consists of analytic functions $f: \mathbb{B} \rightarrow C$ such that

$$
\|f\|_{\mu}=\sup _{z \in \mathbb{B}} \mu(z)|\mathcal{R} f(z)|<\infty,
$$

where $\mu$ is normal.

In [9] and [10], it was shown that $B_{\mu}(\mathbb{B})$ is a Banach space with the norm $\|f\|_{\mathcal{B}_{\mu}}=|f(0)|+\|f\|_{\mu}$.

The little Bloch-type space $B_{\mu, 0}(\mathbb{B})$ consists of analytic functions $f: \mathbb{B} \rightarrow C$ such that

$$
\lim _{|z| \rightarrow 1} \mu(z)|\mathcal{R} f(z)|=0 .
$$

Let $\varphi$ be a holomorphic self-map of $\mathbb{B}$. The composition operator $C_{\varphi}$ as usual is defined by

$$
\left(C_{\varphi} f\right)(z)=(f \circ \varphi)(z), f \in H(\mathbb{B}), z \in \mathbb{B} .
$$

For some results on composition operators, see [2] or [7].

Suppose that $g: \mathbb{B} \rightarrow \mathbb{C}$ is a holomorphic map, define

$$
T_{g} f(z)=\int_{0}^{1} f(t z) \frac{d g(t z)}{d t}=\int_{0}^{1} f(t z) \mathcal{R} g(t z) \frac{d t}{t}, f \in H(\mathbb{B}), z \in \mathbb{B} .
$$

This operator is called the Riemann-Stieltjes operator or extended Cesàro operator, see for example [11].

The Volterra composition operator is defined by

$$
T_{g}^{\varphi} f(z)=\int_{0}^{1} f(\varphi(t z)) \frac{d g(t z)}{d t}=\int_{0}^{1} f(\varphi(t z)) \mathcal{R} g(t z) \frac{d t}{t}, f \in H(\mathbb{B}), z \in \mathbb{B} .
$$

When $\varphi(z)=z$, by (1.1) and (1.2), then $T_{g}^{\varphi} f(z)=T_{g} f(z)$. The Volterra composition operator is a natural extension of the Riemann-Stieltjes or extended Cesàro operator. The Volterra composition operator on the unit disk is considered in [6]. The Volterra composition operators on logarithmic Bloch spaces on $\mathbb{B}$ are studied in [15].

In this paper, we give the characterization of the boundedness and compactness of Volterra composition operator $T_{g}^{\varphi}$ from generally weighted Bloch spaces to Bloch-type spaces. Throughout the remainder of this paper $C$ will denote a positive constant, the exact value of which will vary from one appearance to the next.

\section{The boundedness and compactness of $T_{g}^{\varphi}: \mathcal{B}_{\log }^{\alpha} \rightarrow B_{\mu}$}

In the beginning, we introduce some auxiliary results which will be needed in our proofs of the theorems. 
Lemma 2.1. Let $f \in \mathcal{B}_{\log }^{\alpha}(\mathbb{B})$ and $z \in \mathbb{B}$, then we have

(a) $|f(z)| \leq\left(1+\frac{1}{(1-\alpha) \log 4}\right)\|f\|_{\mathcal{B}_{\log }^{\alpha}}$, when $0<\alpha<1$;

(b) $|f(z)| \leq C\left(\log \log \frac{4}{1-|z|^{2}}\right)\|f\|_{\mathcal{B}_{\log }^{1}}$, when $\alpha=1$;

(c) $|f(z)| \leq(1+A(|z|))\|f\|_{\mathcal{B}_{\text {log }}^{\alpha}}$, when $\alpha>1$,

where $A(|z|)=\int_{0}^{|z|} \frac{d t}{\left(1-s^{2}\right)^{\alpha} \log \frac{4}{1-s^{2}}}$.

Proof. Using the integral representation for $\mathcal{R}$ differential operator, we have

$$
\begin{aligned}
|f(z)| & =\left|f(0)+\int_{0}^{1} \frac{\mathcal{R} f(t z) d t}{t}\right| \\
& \leq|f(0)|+\int_{0}^{1} \frac{|z| d t}{\left(1-|t z|^{2}\right)^{\alpha} \log \frac{4}{1-|t z|^{2}}} \cdot\|f\|_{\mathcal{B}_{\log }^{\alpha}} \\
& \leq|f(0)|+\int_{0}^{|z|} \frac{d s}{\left(1-s^{2}\right)^{\alpha} \log \frac{4}{1-s^{2}}} \cdot\|f\|_{\mathcal{B}_{\log }^{\alpha}} \\
& \leq\|f\|_{\mathcal{B}_{\log }^{\alpha}}+\int_{0}^{|z|} \frac{d s}{\left(1-s^{2}\right)^{\alpha} \log \frac{4}{1-s^{2}}} \cdot\|f\|_{\mathcal{B}_{\log }^{\alpha}} .
\end{aligned}
$$

For $0<\alpha<1, \alpha>1$, then (a) and (c) hold.

For $\alpha=1$,

$$
\begin{aligned}
|f(z)| & \leq\|f\|_{\mathcal{B}_{\log }^{1}}+2 \int_{0}^{|z|} \frac{d s}{(1-s)^{\alpha} \log \frac{4}{1-s}} \cdot\|f\|_{\mathcal{B}_{\log }^{1}} \\
& \leq\left(2 \log \left(2 \log \frac{4}{1-|z|^{2}}\right)+1-2 \log \log 4\right)\|f\|_{\mathcal{B}_{\log }^{1}} \\
& \leq\left(2 \log \log \frac{4}{1-|z|^{2}}+\log 4+1-2 \log \log 4\right)\|f\|_{\mathcal{B}_{\log }^{1}} \\
& \leq C\left(\log \log \frac{4}{1-|z|^{2}}\right)\|f\|_{\mathcal{B}_{\log }^{1}} .
\end{aligned}
$$

Proposition 2.2. Let $\varphi$ be a holomorphic self-map of the open unit ball $\mathbb{B}, g \in H(\mathbb{B})$, and $\alpha>0$. Then $T_{g}^{\varphi}: \mathcal{B}_{\log }^{\alpha}(\mathbb{B}) \rightarrow B_{\mu}(\mathbb{B})$ is compact if and only if for any bounded sequence $\left(f_{j}\right)_{j \in N}$ in $\mathcal{B}_{\log }^{\alpha}(\mathbb{B})$ which converges to zero uniformly on compact subsets of $\mathbb{B}$ as $j \rightarrow \infty,\left\|T_{g}^{\varphi} f_{j}\right\|_{\mathcal{B}_{\mu}} \rightarrow 0$ as $j \rightarrow \infty$.

Proof. Assume that $T_{g}^{\varphi}$ is compact and that $\left(f_{j}\right)_{j \in N}$ is a bounded sequence in $B_{\log }^{\alpha}$ with $f_{j} \rightarrow 0$ uniformly on compact subsets of $\mathbb{B}$. By the compactness of $T_{g}^{\varphi}$, we have that the sequence $\left(T_{g}^{\varphi} f_{j}\right)_{j \in N}$ has a subsequence $\left(T_{g}^{\varphi} f_{j_{m}}\right)_{m \in N}$ which converges to $f$ in $\mathcal{B}_{\mu}$. By Lemma 2.1 and $|f(0)| \leq\|f\|_{B_{\log }^{\alpha}}$, then for any compact $K \subset \mathbb{B}$, there is a $C \geq 0$ such that

$$
\left|T_{g}^{\varphi} f_{j_{m}}(z)-f(z)\right| \leq C\left\|T_{g}^{\varphi} f_{j_{m}}-f\right\|_{\mathcal{B}_{\mu}}, \forall z \in K
$$


This implies that $T_{g}^{\varphi} f_{j_{m}}(z)-f(z) \rightarrow 0$ uniformly on compact subsets of $\mathbb{B}$ as $m \rightarrow \infty$. Since $f_{j_{m}} \rightarrow 0$ on compact subsets of $\mathbb{B}$, by the definition of the operator $T_{g}^{\varphi}$, it is easy to see that for each $z \in \mathbb{B}$, $\lim _{m \rightarrow \infty} T_{g}^{\varphi} f_{j_{m}}(z)=0$. Hence $f=0$. By the arbitrary of $\left(f_{j}\right)_{j \in N}$, we obtain that $T_{g}^{\varphi} f_{j} \rightarrow 0$ in $\mathcal{B}_{\mu}$ as $j \rightarrow \infty$.

Conversely, let $\left\{h_{j}\right\}$ be any sequence in the ball $K_{M}=B_{B_{\log }^{\alpha}}(0, M)$ (at the center of zero with the radius $M$ ) of the space $B_{\log }^{\alpha}$. Since $\left\|h_{j}\right\|_{B_{\log }^{\alpha}} \leq M<\infty$, by Lemma 2.1, $\left\{h_{j}\right\}$ is uniformly bounded on compact subsets of $\mathbb{B}$ and hence normal by Montel's theorem. Hence we may extract a subsequence $\left\{h_{j_{m}}\right\}$ which converges uniformly on compact subsets of $\mathbb{B}$ to some $h \in B_{\log }^{\alpha}$, moreover $h \in B_{\log }^{\alpha}$ and $\|h\|_{B_{\log }^{\alpha}} \leq M$. It follows that $\left(h_{j_{m}}-h\right)$ is that $\left\|h_{j_{m}}-h\right\|_{B_{\log }^{\alpha}} \leq 2 M<\infty$ and converges to zero on compact subsets of $\mathbb{B}$, by the hypothesis, we have that $T_{g}^{\varphi} h_{j_{m}} \rightarrow T_{g}^{\varphi} h$ in $\mathcal{B}_{\mu}$. Thus the set $T_{g}^{\varphi}(K)$ is relatively compact. Hence $T_{g}^{\varphi}: B_{\log }^{\alpha} \rightarrow \mathcal{B}_{\mu}$ is compact.

Here we only consider respectively the following two cases: $0<\alpha<1 ; \alpha>1$. Obviously, $\mathcal{R}\left(T_{g}^{\varphi} f\right)(z)=$ $f(\varphi(z)) \mathcal{R} g(z)$.

Theorem 2.3. Let $\varphi$ be a holomorphic self-map of the open unit ball $\mathbb{B}, g \in H(\mathbb{B}), 0<\alpha<1$. Then the following statements are equivalent.

(i) $T_{g}^{\varphi}: \mathcal{B}_{\log }^{\alpha}(\mathbb{B}) \rightarrow \mathcal{B}_{\mu}(\mathbb{B})$ is bounded;

(ii) $T_{g}^{\varphi}: \mathcal{B}_{\log }^{\alpha}(\mathbb{B}) \rightarrow \mathcal{B}_{\mu}(\mathbb{B})$ is compact;

(iii) $g \in \mathcal{B}_{\mu}$.

Proof. (ii) $\Rightarrow$ (i) By (ii) and the compactness of $T_{g}^{\varphi}: \mathcal{B}_{\log }^{\alpha}(\mathbb{B}) \rightarrow \mathcal{B}_{\mu}(\mathbb{B})$, then (i) holds.

(i) $\Rightarrow$ (iii) By (i), then there exists a positive constant $C$ such that $\left\|T_{g}^{\varphi} f\right\|_{\mathcal{B}_{\mu}} \leq C\|f\|_{\mathcal{B}_{\text {log }}^{\alpha}}$. By taking the test function $f=1$ which is in $\mathcal{B}_{\log }^{\alpha}$, then

$$
\sup _{z \in \mathbb{B}} \mu(z)|\mathcal{R} g(z)| \leq C
$$

Then (iii) holds.

(iii) $\Rightarrow$ (i) For any bounded sequence $\left(f_{k}\right)_{k \in \mathbb{N}}$ in $B_{\log }^{\alpha}$ and $f_{k} \rightarrow 0$ uniformly on compact subsets of $\mathbb{B}$,

$$
\begin{aligned}
\left\|T_{g}^{\varphi} f_{k}\right\| & =\sup _{z \in \mathbb{B}} \mu(z)\left|\mathcal{R}\left(T_{g}^{\varphi} f_{k}\right)(z)\right| \\
& =\sup _{z \in \mathbb{B}} \mu(z)\left|f_{k}(\varphi(z))\right||\mathcal{R} g(z)| \\
& \leq \sup _{z \in \mathbb{B}} \mu(z)\left(1+\frac{1}{(1-\alpha) \log 4}\right)|\mathcal{R} g(z)|\left\|f_{k}\right\|_{\mathcal{B}_{\log }^{\alpha}} \\
& =\sup _{z \in \mathbb{B}} \mu(z)|\mathcal{R} g(z)| \cdot\left\{\left(1+\frac{1}{(1-\alpha) \log 4}\right)\left\|f_{k}\right\|_{\mathcal{B}_{\log }^{\alpha}}\right\} .
\end{aligned}
$$

By (iii), $g \in \mathcal{B}_{\mu}$, moreover, $f_{k} \in B_{\mathrm{log}}^{\alpha}$, then (i) holds.

Theorem 2.4. Let $\varphi$ be a holomorphic self-map of the open unit ball $\mathbb{B}, g \in H(\mathbb{B}), \alpha>1$. Then the following statements are equivalent.

(i) $T_{g}^{\varphi}: \mathcal{B}_{\log }^{\alpha}(\mathbb{B}) \rightarrow \mathcal{B}_{\mu}(\mathbb{B})$ is bounded;

(ii) $g \in \mathcal{B}_{\mu}(\mathbb{B})$ and

$$
\sup _{z \in \mathbb{B}} \mu(z) A(|\varphi(z)|)|\mathcal{R} g(z)|<\infty .
$$


Proof. (ii) $\Rightarrow$ (i) Assume that (ii) holds. Then for $f \in \mathcal{B}_{\log }^{\alpha}$

$$
\begin{aligned}
\left\|T_{g}^{\varphi} f\right\| & =\sup _{z \in \mathbb{B}} \mu(z)\left|\mathcal{R}\left(T_{g}^{\varphi} f\right)(z)\right| \\
& =\sup _{z \in \mathbb{B}} \mu(z)|f(\varphi(z))||\mathcal{R} g(z)| \\
& \leq \sup _{z \in \mathbb{B}} \mu(z)(1+A(|\varphi(z)|))|\mathcal{R} g(z)|\|f\|_{\mathcal{B}_{\text {log }}^{\alpha}}<\infty .
\end{aligned}
$$

By (ii), then we have $T_{g}^{\varphi}: \mathcal{B}_{\log }^{\alpha}(\mathbb{B}) \rightarrow \mathcal{B}_{\mu}(\mathbb{B})$ is bounded.

Conversely, let

$$
\begin{aligned}
& f_{k}(z)=\int_{0}^{\left\langle z, \varphi\left(z_{k}\right)\right\rangle} \frac{d t}{(1-t)^{\alpha} \log \frac{4}{1-t}}, k \in \mathbb{N} \text {, then } f_{k} \in \mathcal{B}_{\text {log }}^{\alpha} . \\
& \infty>\left\|T_{g}^{\varphi} f_{k}\right\|= \sup _{z \in \mathbb{B}} \mu(z)\left|f_{k}(\varphi(z))\right||\mathcal{R} g(z)| \\
& \geq \mu\left(z_{k}\right)\left|f_{k}\left(\varphi\left(z_{k}\right)\right)\right|\left|\mathcal{R} g\left(z_{k}\right)\right| \\
& \geq C \mu\left(z_{k}\right) A\left(\left|\varphi\left(z_{k}\right)\right|\right)\left|\mathcal{R} g\left(z_{k}\right)\right| .
\end{aligned}
$$

Then (2.1) holds. By taking the test function $f=1$ which is in $\mathcal{B}_{\log }^{\alpha}$, then $g \in \mathcal{B}_{\mu}$.

Theorem 2.5. Let $\varphi$ be a holomorphic self-map of the open unit ball $\mathbb{B}, g \in H(\mathbb{B}), \alpha>1$. Then the following statements are equivalent.

(i) $T_{g}^{\varphi}: \mathcal{B}_{\log }^{\alpha}(\mathbb{B}) \rightarrow \mathcal{B}_{\mu}(\mathbb{B})$ is compact;

(ii) $g \in \mathcal{B}_{\mu}(\mathbb{B})$ and

$$
\lim _{|\varphi(z)| \rightarrow 1} \mu(z) A(|\varphi(z)|)|\mathcal{R} g(z)|=0 .
$$

Proof. Assume that $T_{g}^{\varphi}: \mathcal{B}_{\log }^{\alpha} \rightarrow B_{\mu}$ is compact. Let $\left(\varphi\left(z_{k}\right)\right)_{k \in \mathbb{N}}$ be a sequence in $\mathbb{B}$ such that $\left|\varphi\left(z_{k}\right)\right| \rightarrow 1$ as $k \rightarrow \infty$. Let

$$
f_{k}(z)=\left(\int_{0}^{\left\langle z, \varphi\left(z_{k}\right)\right\rangle} \frac{d t}{(1-t)^{\alpha} \log \frac{4}{1-t}}\right)^{2}\left(\int_{0}^{\left|\varphi\left(z_{k}\right)\right|^{2}} \frac{d t}{(1-t)^{\alpha} \log \frac{4}{1-t}}\right)^{-1}, z \in \mathbb{B} .
$$

Then $\left(f_{k}\right)_{k \in \mathbb{N}}$ is a bounded sequence in $\mathcal{B}_{\log }^{\alpha}$ and $f_{k} \rightarrow 0$ uniformly on compact subsets of $\mathbb{B}$,

$$
\left\|T_{g}^{\varphi} f_{k}\right\| \geq \mu\left(z_{k}\right)\left|f_{k}\left(\varphi\left(z_{k}\right)\right)\right|\left|\mathcal{R} g\left(z_{k}\right)\right| \geq C \mu\left(z_{k}\right) A\left(\left|\varphi\left(z_{k}\right)\right|\right)\left|\mathcal{R} g\left(z_{k}\right)\right| .
$$

Then (2.2) holds by letting $k \rightarrow \infty$. By taking the test function $f=1$ which is in $\mathcal{B}_{\log }^{\alpha}$, then $g \in \mathcal{B}_{\mu}$. Conversely, by (2.2), for any given $\varepsilon>0$ there exists a positive number $\delta(\delta<1)$ such that

$$
\mu(z) A(|\varphi(z)|)\left|\mathcal{R} g\left(z_{k}\right)\right|<\varepsilon \text { whenever } \delta<|\varphi(z)|<1 .
$$

For any bounded sequence $\left(f_{k}\right)_{k \in \mathbb{N}}$ in $\mathcal{B}_{\log }^{\alpha}$ and $f_{k} \rightarrow 0$ uniformly on compact subsets of $\mathbb{B}$,

$$
\begin{aligned}
\left\|T_{g}^{\varphi} f_{k}\right\|_{\mathcal{B}_{\mu}}= & \sup _{z \in \mathbb{B}} \mu(z)\left|f_{k}(\varphi(z))\right||\mathcal{R} g(z)| \\
\leq & \sup _{|\varphi(z)| \leq \delta} \mu(z)\left|f_{k}(\varphi(z))\right||\mathcal{R} g(z)| \\
& +\sup _{|\varphi(z)|>\delta} \mu(z)\left|f_{k}(\varphi(z))\right||\mathcal{R} g(z)| \\
\leq & \sup _{|\varphi(z)| \leq \delta} \mu(z)|\mathcal{R} g(z)| \cdot \sup _{|\varphi(z)| \leq \delta}\left|f_{k}(\varphi(z))\right| \\
& +C\left\|f_{k}\right\|_{\mathcal{B}_{\log }^{\alpha}} \sup _{|\varphi(z)|>\delta} \mu(z)|\mathcal{R} g(z)|(1+A(|\varphi(z)|)) .
\end{aligned}
$$

Then $\left\|T_{g}^{\varphi} f_{k}\right\|_{\mathcal{B}_{\mu}} \rightarrow 0$ as $k \rightarrow \infty$. 


\section{Acknowledgements}

This work is supported by the Young Core Teachers Program of Henan Province (No.2011GGJS-062) and the Natural Science Foundation of Henan Province(No.122300410110; 2010A110009).

\section{References}

[1] L. Brown, A. L. Shields. Multipliers and cyclic vectors in the Bloch space, Michigan Math. J. 38(1991), 141-146. 1

[2] C. C. Cowen and B. D. MacCluer. Composition operators on spaces of analytic functions, CRC Press, Boca Roton, 1995. 1

[3] H. Li, P. Liu. Weighted compositioin operators between $H^{\infty}$ and generally weighted Bloch spaces of polydisk. International Journal of Mathematics, 21(5) (2010), 687-699. 1

[4] H. Li, P. Liu. Composition operators between generally weighted Bloch space and $Q_{\log }^{q}$ space, Banach Journal of Mathematical Analysis, 3(1) (2009), 99-110. 1 .

[5] H. Li and X. Yang. Products of integral-type and composition operators from generally weighted Bloch space to $F(p, q, s)$ space, Filomat, 23(3) (2009), 231-241. 1

[6] S. Li. Volterra composition operators between weighted Bergman spaces and Bloch type spaces. J. Korean Math. Soc. 45(1)(2008), 229-248. 1 ]

[7] J. H. Shapiro. Composition operators and classical function theory, Springer-Verlag, New York, 1993. 1

[8] A. L. Shields, D. L. Williams. Bounded proje ctions, duality, and multipliers in spaces of analytic functions, Trans. Amer. Math. Soc. 162 (1971), 287-302. 1

[9] S. Stević. Norm of weighted composition operators from Bloch space to $H_{\mu}^{\infty}$ on the unit ball, Ars. Combin. 88 (2008), 125-127. 1

[10] X. Tang. Extended Cesàro operators between Bloch-type spaces in the unit ball of $\mathbb{C}^{n}$, J. Math. Anal. Appl. 326(2) (2007), 1199-1211. 1

[11] J. Xiao. Riemann-Stieltjes operators between weighted Bloch and Bergman spaces of the unit ball, J. London. Math. Soc. 70(2) (2004), 199-214. 1

[12] S. Ye. Weighted composition operators between different weighted Bloch-type spaces, Acta Mathematica Sinica, Chinese Series, 50(4) (2007), 927-942. 1

[13] R. Yoneda. The composition operator on weighted Bloch space, Arch. Math., 78 (2002), 310-317. 1

[14] K. Zhu. Spaces of Holomorphic functions in the unit ball,Springer, New York, 2005. 1

[15] X. Zhu. Volterra composition operators on logarithmic Bloch spaces. Banach J. Math. Anal. 3(1) (2009), 122-130. 1 